\title{
Editorial: Understanding the Interplay Between Diet, Feed Ingredients and Gut Microbiota for Sustainable Aquaculture
}

\author{
Fotini Kokou ${ }^{1 *}$, Shruti Gupta ${ }^{2}$ and Vikas Kumar ${ }^{3}$ \\ 'Aquaculture and Fisheries, Animal Science Department, Wageningen University, Wageningen, Netherlands, ${ }^{2}$ LetSea, \\ Sandnessjøen, Norway, ${ }^{3}$ Aquaculture Research Institute, Department of Animal, Veterinary and Food Sciences, University of \\ Idaho, Moscow, ID, United States
}

Keywords: fish nutrition, microbiome, aquaculture, fish feed, host-microbe associations, fish health

\section{Editorial on the Research Topic}

\section{OPEN ACCESS}

Edited and reviewed by:

Yngvar Olsen,

Norwegian University of Science and

Technology, Norway

*Correspondence:

Fotini Kokou

fotini.kokou@wur.nl

Specialty section:

This article was submitted to

Marine Fisheries, Aquaculture and

Living Resources,

a section of the journal

Frontiers in Marine Science

Received: 12 January 2022

Accepted: 03 February 2022

Published: 25 February 2022

Citation:

Kokou F, Gupta S and Kumar V (2022)

Editorial: Understanding the Interplay

Between Diet, Feed Ingredients and

Gut Microbiota for Sustainable

Aquaculture.

Front. Mar. Sci. 9:853548

doi: $10.3389 /$ fmars.2022.853548
Understanding the Interplay Between Diet, Feed Ingredients and Gut Microbiota for Sustainable Aquaculture

Aquaculture is one of the fastest-growing food production sectors providing more than half of the fish supply worldwide and thus providing a healthy food source for human consumption. To achieve such growth, aquaculture must overcome several challenges to increase its productivity within our planetary boundaries. These challenges are mainly related to the source and sustainability of its feed ingredients. Traditionally, fish meal and oil have been the main aquafeed ingredients, originating from wild fisheries catch. However, due to the finite nature of these ingredients, materials of plant-origin, such as soybean meal, wheat or corn, as well as novel ingredients such as insect meal, algal meal or microbial proteins, have become an alternative, more sustainable solution, reducing fish-based ingredients in aquafeeds to $<20 \%$ (Turchini et al., 2019; Agboola et al., 2021; Cottrell et al., 2021). To what extent this shift in the nutritional composition will impact the long-term physiology and health of fish, and especially carnivorous fish, is still a subject of extensive research.

Traditionally, research on fish nutrition focused on the phenotypic (i.e., growth, body length), and physiological (i.e., digestion) parameters as a benchmark to evaluate the quality of feed ingredients and diets. However, during the past years, given the vital importance of gut commensal microbes on digestion and health, more studies are addressing the dietary effects on gut microbiota composition in aquatic animals, in addition to the physiological and phenotypic parameters; this may offer complementary information, especially with regard to dietary studies on alternative or novel feed ingredients (Clements et al., 2014; Zhou et al., 2018). Such information will shed light on how alterations in gut microbiota profiles due to different feed ingredient types and diets can be reflected in fish intestinal function, feed efficiency, growth performance and health status (Zhou et al., 2018). Therefore, this Research Topic aimed to gather information on the interaction between dietary formulations and feed ingredients, and the gut microbiota, while evaluating the impact on fish performance, feed utilization, and overall fish health. The Research Topic includes 10 research articles with a general premise on the impact of different dietary ingredients, including pre-and probiotics, on fish performance, health and (gut) microbiota composition. Moreover, the impact of the rearing environment vs. feed was assessed in two of the Research Topic articles.

During the past decades, research on fish meal and oil replacement has been intensive in order to make it possible to switch to alternative feed ingredients. Novel ingredients such as 
microbial meals, insect meals and terrestrial animal by-products have been constantly evaluated on whether they satisfy the demand of the aquafeed industry, with promising applications. Besides growth performance, the impacts of such ingredients on gut health and microbiota composition are of major importance in assessing their value as aquafeed ingredients. In this Research Topic, Tran et al. evaluated the effects of feeding dietary defatted black soldier fly larvae meal on gut health, microbiota and oxidative status of pikeperch. Enhancement of antioxidant activity in the liver, and increase of the microbial richness and diversity in the gut were reported, in agreement with previous studies (Bruni et al., 2018; Huyben et al., 2019), although enrichment of chitin-degrading microbes was not observed (Ringø et al., 2012). Moreover, Solé-Jiménez et al. investigated the impact of microbial and processed animal proteins as the main protein source for gilthead seabream on histology, short-chain fatty acid concentration and microbiota composition in the gut. Total fish meal replacement by this ingredient mixture altered the microbial diversity in the gut along with the short-chain fatty acid concentration. The use of predictive tools to infer microbial functionality indicated that taxa related to inflammatory response increased in abundance when fish meal was replaced. Both studies by Tran et al. and Solé-Jiménez et al. highlighted the connection between the gut health status and microbial markers. Finally, Singh et al. evaluated the use of filamentous fungi as novel alternative ingredients for rainbow trout, as well as the impact of feeding duration and feed processing on the gut microbiota composition and fish performance. The authors reported an increase in lactic acid bacteria in the gut of rainbow trout with fungi addition, which can be a marker for improved gut health.

Nutrient digestion and utilization are among the most important functions of the gut microbiota, affecting also metabolism and growth rate (Llewellyn et al., 2014; Lindsay et al., 2020). Alterations in nutrient composition can alter the microbiota composition; however, the relationship with fish performance is not still well-established. Pelusio et al. evaluated the impact of dietary lipid level and the interaction with different environmental temperatures on the gut microbiota of gilthead seabream, showing that only high temperature affects the microbiota composition. Higher temperatures are known to affect microbiota diversity (Huyben et al., 2018; Kokou et al., 2018); however, an effect was observed only within a few genera, with Lactobacillus prevailing at dietary lipid levels of $16 \%$, and Streptococcus and Bacillus at dietary lipid levels of $21 \%$. Alterations in the type of dietary lipids can also result in gut microbiota differences (Huyben et al., 2020), while such effects can relate to the host's age. Nikouli et al. evaluated the impact of total fish oil replacement by plant oils for Atlantic Salmon postlarvae. The authors reported that the major factor affecting the gut microbiota was the age or developmental stage of the host, with fish from both diets showing a similar microbiota profile. Such findings come in agreement with other studies in early life stages in fish (Xiao et al., 2021), suggesting the importance of comparing similar age groups when examining dietary effects on the gut microbiota.
Besides the main dietary ingredients, prebiotics, probiotics as well as immune-stimulating feed additives are considered important modulators of various biological processes such as digestion, immune stimulation or antioxidative properties through their effects on the gut microbiota (Kiron, 2012; Dawood et al., 2019). Naya-Català et al. evaluated the interaction between fish performance and gut microbiota in gilthead seabream, when egg white hydrolysate was used on plantbased diets. The authors reported that the addition of such peptides improved the gut and liver health when fish were fed plant-based diets, while a change in the gut microbiota was also reported, with proprionate-producing bacteria increasing in abundance. These results suggest that feed additives can potentially restore several negative effects of sub-optimal feed composition via modulation of the gut microbiota. In general, the levels of such additives have to be well-investigated as a negative effect on the growth performance, gut health and microbiota can also be present when included in the diet at high doses. As such, Liu et al. supplemented different levels of histamine in the diets of grouper and reported negative effects when this additive is supplemented in levels $>0.2 \%$. On the other hand, Moroni et al. reported that addition of a nisin-producing probiotic such as the Lactococcus lactis strain, improved the growth intake and immune-related gene expression in gilthead seabream, which also coincided with alteration in the gut microbiota composition. Those positive effects were reported, despite the fact that the probiotic was not able to colonize the gut; this is also supported by other studies (Balcázar et al., 2007; Hoseinifar et al., 2018; Maas et al., 2021a,b), which suggest that probiotic colonization is not necessary to achieve beneficial effects on the gut health and microbiota composition.

Although the feed can be an important factor shaping the gut microbiota, the surrounding environment and environmental factors can also greatly affect the microbiota composition, and thus interfering with results observed by the diet (Giatsis et al., 2015; Xiao et al., 2021). This is more prevalent during early life stages, as Minich et al. reported in yellowtail kingfish, while the microbiota composition can also be differently affected when comparing different tissues. Gills were reported to be more influenced by the surrounding environment than the skin, while the trends in the microbiota diversity are different between external mucosal surfaces like the skin and the gills, and internal surfaces, like the gut, with the latter being also more stable over time. Moreover, in the study by Lorgen-Ritchie et al., the impact of smoltification-the transition from freshwater to seawater-on the gut microbiota of Atlantic salmon was reported, following the same cohort through this process. The transition to seawater had a significant impact on gut microbiota diversity and composition, while there were also distinguishable stage-specific core taxa. Such findings highlight the importance of the rearing environment and life stage on the gut microbiota, which should be considered when studying the effects of diet or feed on the gut microbiota composition.

To conclude, the studies included in this Research topic highlighted the importance of diet, the rearing environment, and 
the feeding trials' duration on the gut microbiota composition. By compiling these ten articles, we hope that researchers and aquaculture professionals in the field of fish nutrition will find this information interesting, especially when developing new feed formulations or testing different feed ingredients and additives. Future studies should connect gut microbiota changes and performance measurements, like growth, gene expression or plasma metabolites. This will enable us to understand how microbiota changes relate to specific phenotypes and provide several valuable biomarkers for fish health and performance. Moreover, exploring the functional properties of the gut microbiota and connecting them to metabolic and physiological

\section{REFERENCES}

Agboola, J. O., Øverland, M., Skrede, A., and Hansen, J. Ø. (2021). Yeast as major protein-rich ingredient in aquafeeds: a review of the implications for aquaculture production. Rev. Aquacult. 13, 949-970. doi: 10.1111/raq.12507

Balcázar, J. L., de Blas, I., Ruiz-Zarzuela, I., Vendrell, D., Calvo, A. C., Márquez, I., et al. (2007). Changes in intestinal microbiota and humoral immune response following probiotic administration in brown trout (Salmo trutta). Br. J. Nutr. 97, 522-527. doi: 10.1017/S0007114507432986

Bruni, L., Pastorelli, R., Viti, C., Gasco, L., and Parisi, G. (2018). Characterisation of the intestinal microbial communities of rainbow trout (Oncorhynchus mykiss) fed with Hermetia illucens (black soldier fly) partially defatted larva meal as partial dietary protein source. Aquaculture 487, 56-63. doi: 10.1016/j.aquaculture.2018.01.006

Clements, K. D., Angert, E. R., Montgomery, W. L., and Choat, J. H. (2014). Intestinal microbiota in fishes: what's known and what's not. Mol. Ecol. 23, 1891-1898. doi: 10.1111/mec.12699

Cottrell, R. S., Metian, M., Froehlich, H. E., Blanchard, J. L., Sand Jacobsen, N., McIntyre, P. B., et al. (2021). Time to rethink trophic levels in aquaculture policy. Rev. Aquacult. 13, 1583-1593. doi: 10.1111/raq.12535

Dawood, M. A., Koshio, S., Abdel-Daim, M. M., and Van Doan, H. (2019). Probiotic application for sustainable aquaculture. Rev. Aquacult. 11, 907-924. doi: $10.1111 /$ raq. 12272

Giatsis, C., Sipkema, D., Smidt, H., Heilig, H., Benvenuti, G., Verreth, J., et al. (2015). The impact of rearing environment on the development of gut microbiota in tilapia larvae. Sci. Rep. 5, 1-15. doi: 10.1038/srep18206

Hoseinifar, S. H., Sun, Y.-Z., Wang, A., and Zhou, Z. (2018). Probiotics as means of diseases control in aquaculture, a review of current knowledge and future perspectives. Front. Microbiol. 9:2429. doi: 10.3389/fmicb.2018.02429

Huyben, D., Roehe, B. K., Bekaert, M., Ruyter, B., and Glencross, B. (2020). Dietary lipid: protein ratio and $\mathrm{n}-3$ long-chain polyunsaturated fatty acids alters the gut microbiome of Atlantic salmon under hypoxic and normoxic conditions. Front. Microbiol. 11:3385. doi: 10.3389/fmicb.2020.589898

Huyben, D., Sun, L., Moccia, R., Kiessling, A., Dicksved, J., and Lundh, T. (2018). Dietary live yeast and increased water temperature influence the gut microbiota of rainbow trout. J. Appl. Microbiol. 124, 1377-1392. doi: 10.1111/jam.13738

Huyben, D., Vidaković, A., Hallgren, S. W., and Langeland, M. (2019). Highthroughput sequencing of gut microbiota in rainbow trout (Oncorhynchus mykiss) fed larval and pre-pupae stages of black soldier fly (Hermetia illucens). Aquaculture 500, 485-491. doi: 10.1016/j.aquaculture.2018.10.034

Kiron, V. (2012). Fish immune system and its nutritional modulation for preventive health care. Anim. Feed Sci. Technol. 173, 111-133. doi: 10.1016/j.anifeedsci.2011.12.015

Kokou, F., Sasson, G., Nitzan, T., Doron-Faigenboim, A., Harpaz, S., Cnaani, A., et al. (2018). Host genetic selection for cold tolerance shapes microbiome composition and modulates its response to temperature. Elife 7:e36398. doi: $10.7554 /$ eLife. 36398

Lindsay, E. C., Metcalfe, N. B., and Llewellyn, M. S. (2020). The potential role of the gut microbiota in shaping host energetics and metabolic rate. J. Anim. Ecol. 89, 2415-2426. doi: 10.1111/1365-2656.13327 changes in the fish-hosts is the next step to improving our understanding of fish-microbe interactions.

\section{AUTHOR CONTRIBUTIONS}

FK prepared the manuscript. SG and VK reviewed the manuscript. All authors approved for publication.

\section{FUNDING}

FK was financially supported by the European Union's Horizon 2020 Reprogram Project (Grant Number 101029186).

Llewellyn, M. S., Boutin, S., Hoseinifar, S. H., and Derome, N. (2014). Teleost microbiomes: the state of the art in their characterization, manipulation and importance in aquaculture and fisheries. Front. Microbiol. 5:207. doi: 10.3389/fmicb.2014.00207

Maas, R. M., Deng, Y., Dersjant-Li, Y., Petit, J., Verdegem, M. C., Schrama, J. W., and Kokou, F. (2021b). Exogenous enzymes and probiotics alter digestion kinetics, volatile fatty acid content and microbial interactions in the gut of Nile tilapia. Sci. Rep. 11, 1-16. doi: 10.1038/s41598-02187408-3

Maas, R. M., Verdegem, M. C., Debnath, S., Marchal, L., and Schrama, J. W. (2021a). Effect of enzymes (phytase and xylanase), probiotics (B. amyloliquefaciens) and their combination on growth performance and nutrient utilisation in Nile tilapia. Aquaculture 533:736226. doi: 10.1016/j.aquaculture.2020.736226

Ringø, E., Zhou, Z., Olsen, R., and Song, S. (2012). Use of chitin and krill in aquaculture-the effect on gut microbiota and the immune system: a review. Aquacult. Nutr. 18, 117-131. doi: 10.1111/j.1365-2095.2011. 00919.x

Turchini, G. M., Trushenski, J. T., and Glencross, B. D. (2019). Thoughts for the future of aquaculture nutrition: realigning perspectives to reflect contemporary issues related to judicious use of marine resources in aquafeeds. North Am. J. Aquacult. 81, 13-39. doi: 10.1002/naaq.10067

Xiao, F., Zhu, W., Yu, Y., He, Z., Wu, B., Wang, C., et al. (2021). Host development overwhelms environmental dispersal in governing the ecological succession of zebrafish gut microbiota. npj. Biofilms Microbiomes 7, 1-12. doi: 10.1038/s41522-020-00176-2

Zhou, Z., Ringø, E., Olsen, R., and Song, S. (2018). Dietary effects of soybean products on gut microbiota and immunity of aquatic animals: a review. Aquacult. Nutr. 24, 644-665. doi: 10.1111/anu.12532

\section{Conflict of Interest: SG was employed by LetSea.}

The remaining authors declare that the research was conducted in the absence of any commercial or financial relationships that could be construed as a potential conflict of interest.

Publisher's Note: All claims expressed in this article are solely those of the authors and do not necessarily represent those of their affiliated organizations, or those of the publisher, the editors and the reviewers. Any product that may be evaluated in this article, or claim that may be made by its manufacturer, is not guaranteed or endorsed by the publisher.

Copyright (c) 2022 Kokou, Gupta and Kumar. This is an open-access article distributed under the terms of the Creative Commons Attribution License (CC BY). The use, distribution or reproduction in other forums is permitted, provided the original author(s) and the copyright owner(s) are credited and that the original publication in this journal is cited, in accordance with accepted academic practice. No use, distribution or reproduction is permitted which does not comply with these terms. 